\title{
Multidrug Resistance Induction
}

National Cancer Institute

\section{Source}

National Cancer Institute. Multidrug Resistance Induction. NCI Thesaurus. Code C40817.

Multidrug Resistance Induction involves initiation of activities of biologic molecules or complexes involved in processes that increase the ability of cancer cells to counteract, defeat, or withstand the effects of multiple chemotherapeutic agents. Mechanisms involve expression of multidrug efflux pumps (p-glycoprotein), altered glutathione metabolism, decreased topoisomerase II activity, and changes in various cellular proteins. 